Effects of Lactobacillus reuteri-containing lozenges on healing after surgical removal of mandibular third molars

\title{
a randomised controlled trial
}

Wälivaara, D.-Å.; Sjögren, I.; Gerasimcik, N.; Yucel-Lindberg, T.; Twetman, S.; Abrahamsson, P.

\section{Published in:}

Beneficial Microbes

DOI:

10.3920/BM2018.0168

Publication date:

2019

Document version

Publisher's PDF, also known as Version of record

Document license:

CC BY-NC-ND

Citation for published version (APA):

Wälivaara, D. -Å., Sjögren, I., Gerasimcik, N., Yucel-Lindberg, T., Twetman, S., \& Abrahamsson, P. (2019).

Effects of Lactobacillus reuteri-containing lozenges on healing after surgical removal of mandibular third molars: a randomised controlled trial. Beneficial Microbes, 10(6), 653-659. https://doi.org/10.3920/BM2018.0168 


\title{
Effects of Lactobacillus reuteri-containing lozenges on healing after surgical removal of mandibular third molars: a randomised controlled trial
}

\author{
D.-Å. Wälivaara ${ }^{1 *}$, I. Sjögren ${ }^{2}$, N. Gerasimcik ${ }^{3}$, T. Yucel-Lindberg ${ }^{3}$, S. Twetman ${ }^{4}$ and P. Abrahamsson $^{1}$ \\ ${ }^{1}$ Maxillofacial Unit, Halland Hospital, 30185 Halmstad, Sweden; ${ }^{2}$ Department of Clinical Microbiology, Halland Hospital, \\ 30185 Halmstad, Sweden; ${ }^{3}$ Division of Periodontology, Department of Dental Medicine, Karolinska Institutet, P.O. Box 4064, \\ 14104 Huddinge, Sweden; ${ }^{4}$ Department of Odontology, Faculty of Health and Medical Sciences, University of Copenhagen, \\ Nørre Allé 20, 2200 Copenhagen N, Denmark; dan-ake.valivaara@regionhalland.se
}

Received: 10 December 2018 / Accepted: 25 April 2019

(c) 2019 Wageningen Academic Publishers

OPEN ACCESS CC) B(1)(-) RESEARCH ARTICLE

\begin{abstract}
We investigated the effect of probiotic supplements on oral wound healing, swelling, pain and discomfort after surgical removal of mandibular third molars. A second aim was to evaluate if the intervention could influence the concentrations of oxytocin in saliva. Sixty-four consecutive volunteers (18-34 years) were enrolled to a double-blind randomised placebo-controlled trial with two parallel arms. Following surgery, the patients were asked to take three lozenges per day containing two strains of Lactobacillus reuteri (DSM 17938 and ATCC PTA 5289) or placebo for two weeks. The clinical healing and extra-oral swelling were scored two weeks post-operatively. Samples of wound exudate were cultivated for the presence of Staphylococcus aureus and $\beta$-haemolytic streptococci. Salivary oxytocin concentrations were analysed from pre- and post-surgery samples using ELISA technique. Compliance and the subjective perception of swelling, pain and discomfort were reported daily through visual analogue scales in a logbook. All patients except three completed the protocol and the postoperative course was uneventful in most cases. Minor extra-oral swellings were noted in five patients, but none required antibiotic treatment. At the 2-week follow-up, there were no significant differences in clinical wound healing index, extra-oral swelling, bacterial growth or salivary oxytocin levels between the groups. The self-reported data unveiled, however, a significantly reduced sense of swelling, in particular during the second week after surgery in the probiotic test group $(P<0.05)$. Likewise, significantly fewer nights with disturbed sleep and fewer days with sick-leave from work were reported among the participants in the test group $(P<0.05)$. No differences were found in the post-operative use of analgesics. In conclusion, we found no significant influence of probiotic supplements on objective wound healing after surgical extraction of impacted mandibular third molars. However, since the patients' perceived significant post-operative ameliorations, further studies are needed to explore the patient's value of the intervention.
\end{abstract}

Keywords: oxytocin, oral wound, probiotics, streptococci

\section{Introduction}

There is emerging evidence that the intake of probiotic bacteria may have beneficial effects on oral health (Twetman et al., 2017). Typically, various lactobacilli strains have been suggested as adjunct to 'good clinical practice' for the prevention and management of childhood caries (Jørgensen et al., 2016), gingivitis and periodontal disease (Gruner et al., 2016), candidiasis (Kraft-Bodi et al., 2015) and halitosis (Keller et al., 2012). It has also been suggested that beneficial bacteria may improve surgical wound healing through a systemic influence on the immune response (Huseini et al., 2012; Jones et al., 2012; Peral et al., 2009; Tsiouris et al., 2017) and via up-regulation of the neuropeptide hormone oxcytocin (Poutahidis et al., 2013). We have previously shown that supernatants of Lactobacillus reuteri can stimulate the production of $\mathrm{PGE}_{2}$ by human gingival fibroblasts in the presence of interluekin-1 $\beta$, and thereby possibly accelerate wound healing (Castiblanco et al., 2017). Furthermore, we noted a tendency to improved healing of 
standardised oral wounds (punch biopsies) during intake of $L$. reuteri supplements (Twetman et al., 2018). Most oral wounds are, however, open sockets, following surgical extractions of teeth. The primary aim of this study was therefore to investigate the effect of probiotic supplements on oral wound healing, swelling, pain and discomfort after surgical removal of mandibular third molars. A second aim was to evaluate if the intervention could influence the local bacterial growth in the wound exudate and the postoperative concentrations of oxytocin in saliva. The null hypothesis was that the wound healing or the perceived swelling, pain and discomfort would not differ from exposure to placebo.

\section{Materials and methods}

\section{Subjects}

The study was carried out between March 2016 and April 2018 at the Oral and Maxillofacial Surgery Department, Halland Hospital in Halmstad, Sweden. Sixty-four consecutive patients (33 females, 31 males) referred for removal of a lower third molar accepted the invitation and were enrolled in the study. The mean age was 29.9 years (range 18-34) and the inclusion criteria were (1) an impacted or partly impacted mandibular third molar with a history of pericoronitis; (2) uncompromised general health; (3) no regular medication (except contraceptives); (4) non-smoker; and (5) living within an area of $40 \mathrm{~km}$ from the hospital. Exclusion criteria were acute pericoronitis and when larger pathological conditions associated with the third molar were detected preoperatively. Eligible patients with regular intake of probiotic supplements prior treatment were not considered for inclusion. The patients received verbal and written information about the study prior to signing the consent form, including their right to end their participation at any time.

\section{Study design and ethical approval}

The study was designed as a single-centre randomised placebo-controlled clinical trial with two parallel arms. The project was ethically approved by the Regional Ethical Committee in Lund, Sweden (Dnr 2015/483) and registered in ClinicalTrials.gov NCT02572531.

\section{Allocation procedure}

After informed written consent, the patients were randomly allocated into two groups receiving either probiotic or placebo lozenges (Figure 1). The randomisation procedure was performed according to a randomisation table. The study was double blind and the lozenges were delivered in identical plastic containers which were separated by a colour code and the code was not unveiled until after the statistical analyses.

\section{Pre-surgical examination and action}

All mandibular third molars were clinically examined before surgery. $60 \%$ were located on the patient's right side and $40 \%$ on the left side. A standard orthopantomography was exposed, supplemented with cone-beam-computertomography, when indicated. A sample of whole mixed saliva was collected through expectoration and stored frozen until further analyses.

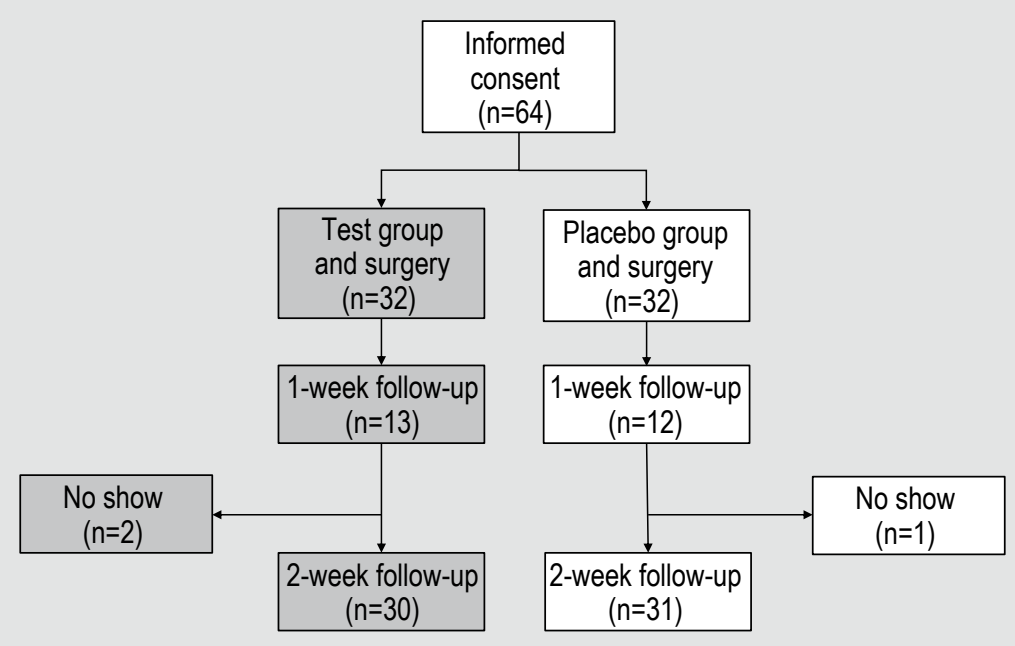

Figure 1. Flow-chart of study participants. Only the first 25 patients enrolled were recalled after 1 week. 


\section{Surgical procedure}

The surgical procedures were performed by six different maxillofacial surgeons in a standardised way. The operating field was anaesthetised with $5.4 \mathrm{ml} 2 \%$ lidocaine with adrenaline. A full thickness mucoperiosteal buccal flap was raised in the region from the lower first molar to the third molar area. To expose the crown to the enamelcement junction of the lower third molar, an osteotomy was performed using a carbon round-bur with a speed of $40,000 \mathrm{rpm}$. The tooth was then separated and removed. The flap was sutured with Vicryl ${ }^{\circ}$ 4-0 sutures (Ethicon, Somerville, NJ, USA) after a thorough debridement and saline rinsing of the operated area. No additional wound dressing was applied.

\section{Postoperative procedures and intervention}

All patients were given standardised postoperative information with recommendations to rinse with $1 \%$ chlorhexidine solution two times per day and take analgesics (paracetamol $1 \mathrm{~g} \times 4$ and ibuprofen $400 \mathrm{mg} \times 3$ ) for the next coming 5 days if and when needed. In addition, the participants were thoroughly instructed to take three lozenges per day containing either two strains of $L$. reuteri (DSM 17938 and ATCC PTA 5289, ProDentis ${ }^{\circ}$, BioGaia $\mathrm{AB}$, Stockholm, Sweden) or placebo for 2 weeks. The active lozenges had at least $2 \times 10^{8}$ live bacteria of the combined strains per lozenge. The placebo lozenges had an identical colour, shape and taste, but without active bacteria. The subjects were told to take one lozenge in the morning, one at lunch time and one in the evening and let it slowly melt in the oral cavity during $5 \mathrm{~min}$. The participants were also provided with a logbook in which the subjective swelling, pain and discomfort were scored with a continuous $0-10$ visual analogue scales (VAS) on a daily basis $(0=$ no swelling/pain/discomfort; 10 = maximum of swelling/ pain/discomfort). Moreover, the use of analgesics, or any other drugs, and any possible perceived side-effects were noted in the same logbook. The VAS were evaluated with a ruler to the nearest 0.5 centimetre. The compliance was also checked through the logbooks; it was considered as 'good' when $>80 \%$ of the lozenges were taken and 'poor' when $<50 \%$ of the lozenges were consumed.

\section{Follow-up}

After 14 days, all patients were recalled for a review appointment in which the individual logbooks were collected. All remaining sutures were removed and the operating field was visually scored with respect to wound healing and possible postoperative infection according to 5-point scale (Table 1) based on Landry et al. (1988). A second whole saliva sample was collected and kept frozen at $-18{ }^{\circ} \mathrm{C}(255 \mathrm{~K})$ until further analyses. From the wound area, a smear sample was collected with aid of sterile tops and transferred for immediate bacterial cultivation. The first 25 patients enrolled in the study were also recalled to a 1-week follow-up for a clinical assessment of the wound healing.

\section{Biochemical and microbial analyses}

After thawing, oxytocin levels, expressed as $\mathrm{pg} / \mathrm{ml}$, were measured using oxytocin ELISA kit (ab133050, Abcam, Cambridge, UK). All steps were followed as instructed by the manufacturer's protocol and a 4-parameter analysis was used to analyse the results. The bacterial samples were investigated for the presence (growth vs non-growth) of Staphylococcus aureus and $\beta$-haemolytic streptococci (Streptococcus pyogenes and Streptococcus dysgalactiae). For S. aureus, a selective agar plate (Staph aureus CHROMagar) was used, in which the bacteria grow with pink colonies. The cultures were incubated at $36{ }^{\circ} \mathrm{C}(309 \mathrm{~K})$ in aerobic conditions for 18-24 h. $\beta$-haemolytic streptococci were evaluated with aid of a two-layered non-selective blood agar plate (Oxoid, Basingstoke, UK) supplemented with $3.5 \%$ horse-blood and $3.5 \%$ sheep-blood. The plates were incubated at $36^{\circ} \mathrm{C}(309 \mathrm{~K})$ in anaerobic conditions for 18 $24 \mathrm{~h}$. The $\beta$-haemolytic colonies were further investigated with Streptococcal latex agglutination (Streptex; Remel Europe Ltd., Dartford, UK).

\section{Table 1. Clinical healing index scores.}

\begin{tabular}{|c|c|}
\hline Healing score & Wound characteristics \\
\hline 5 - excellent & All wound area is pink; no bleeding on palpation; no granulation tissue; no connective tissue at incision margin. \\
\hline 4 - very good & $\begin{array}{l}\text { Less than one quarter of wound area is red, no bleeding on palpation, no granulation tissue; no connective tissue at incision } \\
\text { margin. }\end{array}$ \\
\hline $3-$ good & Less than half of the wound area is red; no bleeding on palpation; no granulation tissue; no connective tissue at incision margin. \\
\hline $2-$ poor & More than half of the wound area is red; bleeding on palpation; granulation tissue evident; incision margin not epithelialised. \\
\hline 1 - very poor & $\begin{array}{l}\text { Two or more of the following features: More than half of the wound area is red; bleeding on palpation; granulation tissue evident; } \\
\text { no epithelium at incision margin; infection present with suppuration. }\end{array}$ \\
\hline
\end{tabular}




\section{Statistical methods}

All data were processed with the IBM SPSS software (version 23.0, Armonk, NY, USA). For continuous data, the follow-up values were compared to baseline within each group by Wilcoxon paired two-sided test and differences between groups are analysed by Wilcoxon unpaired test. Categorical scores were compared between groups with chi-square tests. $P$-values less than 0.05 were considered statistically significant. The sample size was estimated with aid of the MedCalc software (Ostend, Belgium) and it was anticipated that a $30 \%$ difference in healing, pain and discomfort would be of clinical importance. With $\alpha=0.05$ and $\beta=0.2$, the power calculation indicated that at least 30 subjects were needed in each group to disclose a statistically significant difference between the groups.

\section{Results}

\section{Clinical observations}

Three patients dropped out from the protocol as they failed to show up for the 2-week examination and thus, no logbooks were received. The clinical healing was uneventful in most cases. Minor extra-oral swellings were noted in five patients, but none required antibiotic treatment. The distribution of the clinical healing index is shown in Table 2. At the 2-week follow-up, there were no differences between the groups. However, when the 25 patients that attended the 1-week were analysed separately, the distribution of the healing scores was in favour for the probiotic intervention group.

\section{Biochemical and microbial evaluation}

The pre- and post-treatment concentrations of salivary oxytocin are shown in Table 3. There were no significant differences between the groups and the levels remained also fairly stable within the groups, albeit a weak tendency of reduced concentrations was noted in the placebo group. Growth of S. aureus was detected in seven patients from the test group and six patients in the control group and presence of $\beta$-haemolytic streptococci was recorded in three and four patients, respectively.

\section{Self-reported data and compliance}

The self-reported perception of swelling, pain and discomfort are summarised in Table 4 and the data on fever, sleep, analgesics and sick-leave are shown in Table 5. There were no significant differences between the groups with few exceptions; the sense of swelling during the second week after surgery was significantly lower $(P<0.05)$ in the probiotic test group as illustrated day-by-day in Figure 2. Likewise, significantly fewer nights with disturbed sleep and fewer days with sick leave from work $(P<0.05)$ were reported among the participants of the test group (Table $5)$. The compliance with the protocol was rated as good in

Table 2. Distribution of clinical healing score after 1 and 2 weeks after the third-molar surgery.

\begin{tabular}{llllll} 
Time and group & \multicolumn{2}{l}{ Healing score (\%) } & & & \\
\cline { 2 - 6 } & 1 & 2 & 3 & 4 & 5 \\
& & & 31 & $31^{\text {a }}$ & - \\
1 week - test group $(n=13)$ & 15 & 23 & 42 & 42 & - \\
1 week - placebo group $(n=12)$ & - & 17 & 7 & 40 & 63 \\
2 weeks - test group $(n=30)$ & - & - & 7 & 32 & 61 \\
2 weeks - placebo group $(n=31)$ & - & - & & \\
\hline
\end{tabular}

a Significant difference in distribution compared with placebo (chi-square-test, $P<0.05$ ).

Table 3. Salivary concentrations $(\mathrm{pg} / \mathrm{ml})$ of oxytocin at baseline and at the 2-week follow-up. ${ }^{1}$

$\begin{array}{llll}\text { Time } & \text { Test group }(\mathrm{n}=\mathbf{3 0}) & \text { Placebo group }(\mathrm{n}=31) & P \text {-value } \\ \text { Baseline } & 107.4(24.3) & 90.9(15.6) & 0.56(\mathrm{NS})^{2} \\ \text { 2-week follow-up } & 106.8(22.5) & 81.7(16.6) & 0.37 \text { (NS) } \\ \text { Mean difference } & 0.7(17.0) & 9.3(19.0) & 0.61 \text { (NS) }\end{array}$

${ }^{1}$ Values in the table denote mean and standard error.

${ }^{2} \mathrm{NS}=$ not statistically significant. 
Table 4. Self-reported swelling, pain and discomfort during the first and second week after third molar surgery. ${ }^{1}$

\begin{tabular}{|c|c|c|c|}
\hline Time and group & Swelling & Pain & Discomfort \\
\hline $1^{\text {st }}$ week - test group $(n=13)$ & $19.4(9.8)$ & $25.0(12.9)$ & $23.0(11.9)$ \\
\hline $1^{\text {st }}$ week - placebo group $(n=12)$ & $23.9(17.3)$ & $26.1(16.2$ & $24.9(16.2)$ \\
\hline $2^{\text {nd }}$ week - test group $(n=30)$ & $2.8(3.8)^{2}$ & $9.9(12.3)$ & $6.6(7.9)$ \\
\hline $2^{\text {nd }}$ week - placebo group $(n=31)$ & $8.9(10.9)$ & $12.3(13.8)$ & $10.0(11.3)$ \\
\hline
\end{tabular}

Table 5. Self-reported data on postoperative fever, sick-leave and consumption of analgesics. ${ }^{1}$

\begin{tabular}{llll} 
Measure & Test group (n=30) & Placebo group (n=31) & $P$-value \\
Fever, yes (\%) & 79 & 83 & 0.69 (NS) \\
Fever, number of days (mean, sd) & $0.4(0.9)$ & $0.4(0.9)$ & 1.00 (NS) \\
Disturbed sleep, yes (\%) & 42 & 61 & 0.14 (NS) \\
Disturbed sleep, number of nights (mean, sd) & $1.5(2.4)$ & $2.9(2.8)$ & $<0.05^{3}$ \\
Analgesics 1st week, number of tablets (mean, sd) & $34.0(22.1)$ & $39.3(21.8)$ & 0.35 (NS) \\
Analgesics 2 ${ }^{\text {nd }}$ week, number of tablets (mean, sd) & $12.4(18.6)$ & $8.1(13.4)$ & 0.30 (NS) \\
Sick leave, number of days (mean, sd) & $0.3(0.5)$ & $1.1(1.5)$ & $<0.05^{3}$ \\
\hline${ }^{1}$ Values are percent or mean with standard deviations (sd). & & & \\
${ }^{2}$ NS = not statistically significant. & & & \\
${ }^{3}$ Statistically significant difference compared with placebo, independent sample t-test. & &
\end{tabular}

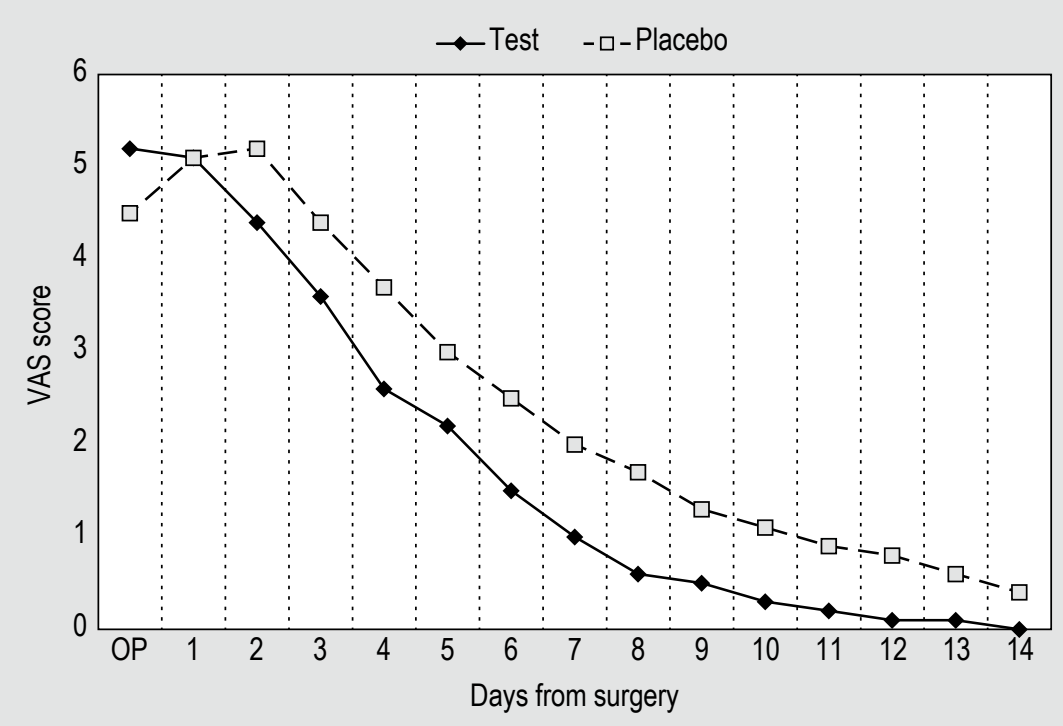

Figure 2. Patients' day-by-day perception of oral swelling during the first two weeks after mandibular $3^{\text {rd }}$ molar surgery with and without probiotic supplements. The values denote mean visual analogue scales (VAS) score (0-10). The maximal standard deviation was on day one (2.6) and the minimum on day $14(0.2)$. 
over $70 \%$ of the patients in both groups. Poor compliance was found among $19 \%$ of the patients in the test group and $11 \%$ in the placebo group.

\section{Discussion}

To our best knowledge, this is the first study in which the use of probiotics supplements has been evaluated in maxillofacial surgery. The reason to select $L$. reuteri was that one of the strains was isolated from the oral cavity and that this consumer product has proven beneficial effects on various oral conditions (Twetman et al., 2017). The main finding was that no significant difference in the clinical wound healing score was disclosed between the groups and that no major complications or secondary infections were present in neither of the groups. The null hypothesis could, however, not be completely rejected as some interesting results from the self-reported endpoints were obtained. The patients in the test group perceived less post-operative swelling and reported significantly less nights with affected sleep and fewer days with sick leave. Such perceived reliefs are certainly important and of value for the individual patient, but we found no specific pattern or correlations to gender or age. It was interesting to find that most patients followed the recommended intake of analgesics during the first week with an average of 7-8 tablets per day, although it was emphasised that the use should be restricted to the individual need. In contrast, only $47 \%$ of the patients used chlorhexidine rinses and they were equally distributed in the test and the placebo group. Notably, a 'per protocol' analysis based selectively on those with good compliance did not alter any of the results in a significant way.

There are a number of limitations to be considered. First of all, the clinical healing was scored two weeks after the operation and an earlier follow-up would probably have been more discriminating. In fact, a one-week examination was originally planned but this was pragmatically abandoned since the frequent recall-schedule was a severe obstacle in the enrolment process. Thus, only the first 25 patients were examined one week postoperatively and interestingly, a clear trend in favour for the probiotic supplements were seen. Second, the intervention was commenced in connection with the surgery and it is possible that an intake of probiotic supplements 1-2 weeks prior to surgery could have amended the results, as it may take some day to initiate the immune response. Third, the wound healing index applied here was originally suggested for periodontal surgery and has to our knowledge not previously been applied for mandibular third molar surgery. A fourth fact to consider is that the surgical procedure was carried out by a number of different oral surgeons and it is well known that the rate of success and failures can vary with the operators experience and skills (Del Fabbro and Taschieri, 2007). Furthermore, the use of chlorhexidine mouth rinses may have influenced the results. Since the use of such antibacterial is a recommended procedure after oral surgery, we had to retain the rinses in this project for ethical reasons as 'best clinical practice.' On the other hand, the low compliance and equal distribution between the groups reduced the risk of bias. Finally, the self-reported data were for natural reasons not validated. The participants were strongly emphasised to fill in the logbook on a daily basis in order to minimise recall bias but partly incomplete data sets were seen in almost $40 \%$ of the questionnaires. The large inter-individual variations in the VAS scores reflected certainly considerable differences in the patient's subjective perception of pain, swelling and discomfort.

The reason for analysing the growth of S. aureus and beta-haemolytic streptococci was that those species often are involved in wound infections and impaired healing (Hughes et al., 2009; Tong et al., 2015). Mirroring the noncomplicated clinical findings, we found a low prevalence in both groups. Furthermore, the patients with growth displayed low to moderate numbers of colony forming units and only one patient exhibited abundant growth of S. aureus. It should, therefore, be underlined that present findings cannot be generalised to the situation of chronic oral wounds often appearing among immunosuppressed patients and frail elderly. The non-affected concentrations of salivary oxytocin were not supportive to the study of Poutahidis and co-workers (2013), but in agreement with our previous pilot observations (Twetman et al., 2018).

\section{Conclusions}

Within the limitations of the present study, we found no significant influence of probiotic supplements on oral wound healing after surgical extraction of impacted mandibular third molars. The patients reported, however, less swelling during the second week after operation and fewer days with sick-leave and fewer nights with disturbed sleep. Further studies are however needed to further explore the patient's perceived value of the intervention. In particular, more frequent follow-ups and an extended intervention, starting well ahead of the surgery, would be of interest.

\section{Acknowledgements}

The authors would like to thank BioGaia AB, Lund, Sweden for providing the lozenges for the study.

\section{Conflicts of interest}

Svante Twetman has received travel support from BioGaia $\mathrm{AB}$ for lectures and symposium on probiotic therapy at dental congresses. The other authors have no conflict of interest to declare. 


\section{References}

Castiblanco, G., Yucel-Lindberg, T., Roos, S. and Twetman, S., 2017. Effect of Lactobacillus reuteri on cell viability and PGE2 production in human gingival fibroblasts. Probiotics and Antimicrobial Proteins 9: 278-283.

Del Fabbro, M. and Taschieri, S., 2007. A systematic review on the outcome of surgical vs. non-surgical procedure for the retreatment of periapical lesions. Minerva Stomatologia 56: 621-632.

Gruner, D., Paris, S. and Schwendicke, F., 2016. Probiotics for managing caries and periodontitis: systematic review and meta-analysis. Journal of Dentistry 48: 16-25.

Hughes, J.M., Wilson, M.E., Brandt, C.M. and Spellerberg, B., 2009. Human infections due to Streptococcus dysgalactiae subspecies equisimilis. Clinical Infectious Disseases 49: 766-772.

Huseini, H.F., Rahimzadeh, G., Fazeli, M.R., Mehrazma, M. and Salehi, M., 2012. Evaluation of wound healing activities of kefir products. Burns 38: 719-723.

Jones, M., Ganopolsky, J.G., Labbé, A., Gilardino, M., Wahl, C., Martoni, C. and Prakash, S., 2012. Novel nitric oxide producing probiotic wound healing patch: preparation and in vivo analysis in a New Zealand white rabbit model of ischaemic and infected wounds. International Wound Journal 9: 330-343.

Jørgensen, M.R., Castiblanco, G., Twetman, S. and Keller, M.K., 2016. Prevention of caries with probiotic bacteria during early childhood. Promising but inconsistent findings. American Journal of Dentistry 29: 127-131.

Keller, M.K., Bardow, A., Jensdottir, T., Lykkeaa, J. and Twetman, S., 2012. Effect of chewing gums containing the probiotic bacterium Lactobacillus reuteri on oral malodour. Acta Odontologica Scandinavica 70: 246-250.
Kraft-Bodi, E., Jørgensen, M.R., Keller, M.K., Kragelund, C. and Twetman, S., 2015. Effect of probiotic bacteria on oral candida in frail elderly. Journal of Dental Research 94, Suppl. 9: 181S-186S.

Landry, R.G., Turnbull, R.S. and Howley, T., 1998. Effectiveness of benzydamyne $\mathrm{HCl}$ in the treatment of periodontal post-surgery patients. Research in Clinic Forums 10: 105-118.

Peral, M.C., Martinez, M.A. and Valdez, J.C., 2009. Bacteriotherapy with Lactobacillus plantarum in burns. International Wound Journal 6: 73-81.

Poutahidis, T., Kearney, S.M., Levkovich, T., Qi, P., Varian, B.J., Lakritz, J.R., Ibrahim, Y.M., Chatzigiagkos, A., Alm, E.J. and Erdman, S.E., 2013. Microbial symbionts accelerate wound healing via the neuropeptide hormone oxytocin. PLoS ONE 8: e78898.

Tong, S.Y., Davis, J.S., Eichenberger, E., Holland, T.L. and Fowler, V.G., 2015. Staphylococcus aureus infections: epidemiology, pathophysiology, clinical manifestations, and management. Clinical Microbiological Reviews 28: 603-661.

Tsiouris, C.G., Kelesi, M., Vasilopoulos, G., Kalemikerakis, I. and Papageorgiou, E.G. 2017. The efficacy of probiotics as pharmacological treatment of cutaneous wounds: meta-analysis of animal studies. European Journal of Pharmaceutical Sciences 104: 230-239.

Twetman, S., Jørgensen, M.R. and Keller, M.K., 2017. Fifteen years of probiotic therapy in the dental context - what has been achieved? Journal of the Californian Dental Association 45: 539-545.

Twetman, S., Keller, M.K., Lee, L., Yucel-Lindberg, T. and Pedersen, A-M.L., 2018. Effect of probiotic lozenges containing Lactobacillus reuteri on oral wound healing: a pilot study. Beneficial Microbes 9: 691-696. 
